\title{
1011 A TRIP UNPLANNED: AN ATV SAFETY STORY
}

Injury Prevention Center at Arkansas Children's Hospital, funded by EMSC Targeted Issues Grant program Litzwire Sound + Vision Correspondence to University of Arkansas for Medical Sciences, Pediatrics/CARE Slot 512-26 1 Children's Way Little Rock, Arkansas 72202, USA

\subsection{6/ip.2010.029215.1011}

All-terrain vehicle (ATV) injury is a common and growing problem among children and adolescents, particularly in rural areas. In 2007 alone, US children under 16 years old sustained an estimated 40000 ATV-related injuries that required treatment in emergency departments, and children accounted for $20 \%$ of ATV deaths and $27 \%$ of all injuries. Most paediatric ATV deaths occur when they are riding adult-sized vehicles and often involve other risky behaviour, such as lack of helmet use and riding with passengers. All-terrain vehicles are commonly used in outdoor recreational activities, including hunting, along with farming and other agricultural work. Safety education targeting youths who frequently ride ATVs in these settings may be an effective means of reducing risk for ATVrelated injury. Previous qualitative research demonstrated that such educational interventions must be compelling, age appropriate, and realistic to engage the target audience. A trip unplanned: an ATV safety story is a 22 min video dramatisation that illustrates the consequences of ATV use and provides viewers strategies to reduce the risk of serious injury. The film is designed to appeal to an adolescent audience and its content is appropriate for use both in school settings and in hunter safety education programs. Since such programs are often mandated as part of hunter licensing, it is important to have accurate and compelling educational tools such as this video to reach this audience, who are likely to be exposed to ATVs as part of their hunting recreational activities. Audiences exposed to the video have demonstrated an increased knowledge of recommended ATV riding behaviours to reduce risks, including use of helmets and other safety gear, safe firearm transportation, and having no riders except for the operator or driver. The video forms part of a larger 'toolkit' of thematically-related educational materials. 